\title{
Fecal Lactoferrin Predicts Primary Nonresponse to Biologic Agents in Inflammatory Bowel Disease
}

\author{
Dario Sorrentino ${ }^{\mathrm{a}, \mathrm{b}}$ Vu Q. Nguyen ${ }^{\mathrm{a}}$ Kim Love $^{\mathrm{c}}$ \\ a IBD Center, Division of Gastroenterology, Virginia Tech Carilion School of Medicine, Roanoke, VA, USA; \\ ${ }^{b}$ Department of Clinical and Experimental Medical Sciences, University of Udine School of Medicine, Udine, Italy; \\ 'K.R. Love Quantitative Consulting and Collaboration, Athens, GA, USA
}

\section{Keywords}

Biomarkers · Inflammatory bowel diseases · Treatment response $\cdot$ Fecal lactoferrin · Biologics

\begin{abstract}
Introduction: Fecal lactoferrin (FL) is a timely and accurate marker of inflammation in ulcerative colitis (UC) and Crohn's disease (CD). The aim of this study was to verify whether FL can predict primary nonresponse (PNR) to biologic agents during induction. Methods: Retrospective outcome review in 27 patients ( 13 with CD and 14 with UC) tested for baseline FL and retested within a week after the first and second induction doses. Clinical/biochemical outcomes were evaluated at end of induction and at follow-up (3-24 months). $R \boldsymbol{e}$ sults: Compared to baseline, changes of the Harvey-Bradshaw (CD) and Partial Mayo Scoring (UC) indices at end of induction separated responders (18/27 or 67\%) from nonresponders (9/17 or $33 \%)$. In all patients, the initial FL value at induction decreased compared to baseline, continuing to decrease after the following dose in clinical responders while bouncing back in the others. Models targeting the 2 consecutively decreased FL values or the second FL value compared to baseline or the second FL value compared to the first were able to accurately predict response at end of induction. Follow-up assessment confirmed clinical remission in
\end{abstract}

karger@karger.com www.karger.com/ddi

Karger!"

GOPEN ACCESS
(C) 2021 The Author(s)

Published by S. Karger AG, Basel

This is an Open Access article licensed under the Creative Commons Attribution-NonCommercial-4.0 International License (CC BY-NC) (http://www.karger.com/Services/OpenAccessLicense), applicable to the online version of the article only. Usage and distribution for commercial purposes requires written permission. initial responders (with FL values reduced on the average by $94 \pm 10 \%$ compared to baseline). Conclusions: In CD and UC patients during induction with biologic agents, early FL measurements accurately separate clinical responders from those experiencing PNR. The method described here offers several potential advantages over other strategies to assess and manage these patients.

(C) 2021 The Author(s)

Published by S. Karger AG, Basel

\section{Introduction}

Primary response to biologic induction in inflammatory bowel disease (IBD) - both ulcerative colitis (UC) and Crohn's disease (CD) - has been traditionally evaluated by the attenuation of clinical symptoms after 12 weeks $[1,2]$. More recently, the measurement of drug trough levels (TL) and of antidrug antibodies (ADA), a strategy known as therapeutic drug monitoring (TDM), has been used to evaluate possible loss of response (LOR) [3] and proposed by some to proactively reduce primary nonresponse (PNR) and LOR incidence [4-6]. Although proactive TDM-based management might improve outcomes in IBD [4-6], this strategy is expensive and has practical shortcomings [7-11] and it is unclear whether it

Correspondence to:

Dario Sorrentino,drsorrentino@ carilionclinic.org 
is truly superior to reactive TDM or to the clinically based management $[12,13]$. Ideally, it is the individual patient disease burden that should be targeted by therapy [1416]. Fecal lactoferrin (FL) and calprotectin (FC) are accurate indicators of intestinal mucosa inflammation [17], but their role in the management of therapeutic response is still evolving [18]. A necessary feature of any strategy for the management of LOR and PNR is the test time sensitivity, that is, the immediate responsiveness to drug-induced changes in mucosa inflammation. We have published evidence that FC levels in IBD patients on biologics timely and sensibly reflect the increasing inflammatory activity during the therapeutic interval due to the progressive neutralization of the medication [19]. Indeed, fecal marker levels in stool are directly related to neutrophil translocation to the mucosa of the GI tract - a process quickly modulated by medications acting on the activity of the inflammatory process [20]. We have since leveraged on those findings to routinely evaluate the immediate response to treatment in patients undergoing biologic induction. In this retrospective study, we report the relevant data.

\section{Methods}

\section{Study Design and Study Population}

This retrospective study enrolled 27 consecutive patients with UC or CD diagnosed and staged according to established criteria [21], to be treated with biologic monotherapy (infliximab, adalimumab, certolizumab, and vedolizumab) as standard of care in our IBD center and enrolled within a 2-year period (2018-2020). No steroids or other medications were prescribed before the index FL tests and the end of induction. All patients tested negative for tuberculosis and hepatitis $\mathrm{B}$ and $\mathrm{C}$ as well as stool culture and $\mathrm{C}$. difficile.

FL levels as well as clinical/laboratory data were collected from the patients' electronic medical records. Data collected involved patients (both naïve and biologic experienced) undergoing biologic induction. In these patients, FL was measured at baseline (within 1 month of starting therapy), then within a week after drug administration for at least 2 consecutive infusions/injections during the induction phase (comprising 3 infusions for infliximab and vedolizumab [weeks 0,2 , and 6], 2 injections for adalimumab [weeks 0 and 2], and 3 injections for certolizumab [weeks 0, 2, and 4]). Management of these patients was based on clinical outcomes and scores (see below) after completion of the induction phase. Followup was 3-24 months - on the average 8 months - and included clinical and biochemical (postinduction FL levels) assessment.

\section{Biomarker Testing}

FL was measured in samples collected during the first bowel movement of the day by the LACTOFERRIN SCAN (TECHLAB, Blacksburg, VA, USA), an ELISA. Values $\leq 7.24 \mu \mathrm{g} / \mathrm{mL}$ are considered normal [22]. At Carilion Clinic Laboratory, the lower limit to which the linearity of results has been validated is $6.25 \mu \mathrm{g} / \mathrm{mL}$, which is the absolute value reported and analyzed for results $\leq 6.25$ $\mu \mathrm{g} / \mathrm{mL}$. FL values are presented by standard decimal approximations of the actual number by excess or defect. Routine blood and stool studies were performed using established methods.

\section{Assessment of Clinical Disease Activity}

Clinical assessment was performed during clinic visits at baseline, within a week of completing induction and at follow-up and was based on the Harvey-Bradshaw Index (HBI) for CD and the Partial Mayo Scoring Index (PMSI) for UC [23]. For HBI, scores of $<5,5-7,8-16$, and $>16$ define remission, mild, moderate, and severe disease activity. The same activities are defined by the scores of 0-1, 2-4, 4-6, and 7-9 for PMSI. Clinical response in UC was defined as a decrease of $\geq 3$ of the partial Mayo score [24] plus a decrease of at least $30 \%$ from the baseline score, accompanied by a decrease of $\geq 1$ in the rectal bleeding scale or an absolute rectal bleeding score of 0 or 1 [25]. Response in CD was defined as a decrease of $\geq 3$ of the HBI [26].

\section{Statistical Analysis}

Patients were separated in 2 groups based on the postinduction clinical response. To uniformly analyze HBI and PMSI, these indices were rescaled to be between 0 and 1 by dividing each score by the maximum value for each scale with the value of " 16 " used as the maximum value for the HBI since all the scores above 16 are considered severe (and 16 was also the highest value of the $\mathrm{HBI}$ in the data set). Analysis of these rescaled HBI and PMSI before and after induction treatment was performed using a nonparametric sign test within each group due to nonnormality of these values; a Mann-Whitney U test was used to compare baseline values. We also used nonparametric Mann-Whitney $U$ test to compare the baseline FL values and the initial FL drop in the 2 groups of patients.

Various combinations of FL measures were used to predict whether an individual would respond clinically (based on the criteria outlined in the previous section) following the entire induction cycle. The first of these measures was a dichotomous measure where participants were labeled as FL responders if they experienced 2 consecutive drops in FL compared to their baseline measurement and labeled as FL nonresponders if they did not. The remaining measures were all numeric measures of FL (generally based on $\%$ of baseline measure), including FL values following the first induction dose (as a percentage of baseline values), FL values following the second induction dose (as a percentage of baseline values), the baseline FL measure itself (in raw units), and the difference between the first and the second FL values (as a percentage of the baseline value).

Logistic regression models were used to examine the relationships of each of these predictors to the outcome of remission. For all models, area under the receiver operating characteristic curve (AUC), accuracy, sensitivity, specificity, and FL cutoff values were calculated.

\section{Results}

There were 27 patients who underwent biologic induction (Table 1) with $14 / 27$ being biologic naïve. The median age was 51 years, $48 \%$ had CD (with approxi- 
Table 1. Patient features

\begin{tabular}{ll}
\hline Demographics & $N=27$ \\
Age, median (IQR), years & $51(32.5)$ \\
Males-females & $15-12$ \\
Smoker (current, former, and never), \% & 18,30 , and 52 \\
\hline Disease characteristics & \\
Disease type & \\
CD, $N(\%)$ & $13(48)$ \\
UC, $N(\%)$ & $14(52)$ \\
Disease duration, median (IQR), years & $6(8)$ \\
Biologic naïve (yes/no) & $14 / 13$ \\
Biologic induction & \\
Infliximab, $n$ (\%) & $15(55)$ \\
Vedolizumab, $n(\%)$ & $7(30)$ \\
Adalimumab, $n$ (\%) & $4(15)$ \\
Certolizumab, $n(\%)$ & $1(4)$ \\
CD location, $\%$ & \\
L1: ileal & 30 \\
L2: colonic & 30 \\
L3: ileocolonic & 40 \\
CD behavior, \% & \\
B1: nonstricturing, nonpenetrating & 85 \\
B2: stricturing & 15 \\
B3: penetrating & 0 \\
UC location, $\%$ & \\
E1: ulcerative proctitis & 15 \\
E2: left sided (distal) & 21 \\
E3: extensive (pancolitis) & \\
\hline
\end{tabular}

CD, Crohn's disease; UC, ulcerative colitis.

mately equally distributed ileal, ileocolonic, and colonic disease), and $52 \%$ had UC (2/3 with pancolitis). In CD patients the disease was mostly nonstricturing/nonpenetrating.

Of the 27 patients, $18(67 \%)$ responded clinically to induction with normalized clinical scores dropping from $0.71 \pm 0.16$ to $0.05 \pm 0.06,(p<0.0001)$ (Table 2$)$. In all cases the numerical value of both HBI and PMSI fell within the definition of remission. In 9/27 (33\%) patients, normalized clinical scores remained unchanged after induction ( $0.79 \pm 0.19$ vs. $0.71 \pm 0.13, p=0.06)$. Baseline clinical score values were very similar among the 2 groups $(p=0.28)$. Although FL substantially decreased (by up to $98 \%$ of baseline levels) in all patients after the first infusion/injection, subsequent FL levels further decreased (or stayed within normal values/did not increase) only in clinical responders during induction (Table 2). In clinical nonresponders, subsequent FL levels increased, in some cases reaching levels close or superior to baseline values (Table 2). The data for all patients are graphically reported in Figure 1. No changes in management were made in the first group of patients since they responded to induction. However, the patients in the second group continued to experience severe symptoms after induction and were reinduced with other biologics or underwent surgery if they had failed multiple biologics.

The baseline FL values were greater in the nonresponders group compared to responders $(2,221 \pm 1,910$ vs. $773 \pm 1,054 \mu \mathrm{g} / \mathrm{mL}, p=0.02)$. Likewise, the initial FL drop was less on the average in the nonresponders although the difference was not statistically significant (62 \pm 36 vs. $83 \pm 16 \%, p=0.09$ ).

Diagnostic accuracy for FL in predicting clinical response was calculated for different models (see Methods). Using the drop in FL values after the first induction dose (as a percentage of the baseline FL), the AUC associated with the receiver operating curve is 0.710 with a $95 \%$ CI of 0.497-0.923 (see online suppl. Fig. 1; see www.karger. com/doi/10.1159/000515432 for all online suppl. material). The FL cutoff (probability of clinical response $>0.5$ ) is $45.6 \%$ of baseline with sensitivity of 1 and $95 \%$ CI of $0.847-1$ and specificity of 0.333 and $95 \%$ CI of $0.075-$ 0.701 . Using the drop in FL value after the second drug dose (as a percentage of baseline FL), the AUC is estimated to be 1 (online suppl. Fig. 2). In this case, the FL cutoff is $42.7 \%$ of baseline with a sensitivity of 1 ( $95 \% \mathrm{CI}=0.847$, 1 ) and specificity of $1(95 \% \mathrm{CI}=0.717,1)$. Baseline FL ( $\mu \mathrm{g} /$ $\mathrm{mL}$ ) was also able to predict clinical response at end of induction with AUC of 0.778 (95\% CI $=0.570-0.986)$, sensitivity of $0.762(95 \% \mathrm{CI}=0.528,0.918)$, specificity of $0.444(95 \% \mathrm{CI}=0.137,0.788)$, and baseline FL cutoff of $2,334.07 \mu \mathrm{g} / \mathrm{mL}$ (online suppl. Fig. 3). Using the change in FL value after the second drug dose compared to the value after the first drug dose, the AUC is estimated to be 1 with a sensitivity of $1(95 \% \mathrm{CI}=0.847,1)$, a specificity of $1(95 \% \mathrm{CI}=0.717,1)$, and a FL cutoff of $25 \%$ of baseline FL (online suppl. Fig. 4). Finally, using the 2 consecutive FL drops as a criterion to predict remission, the AUC is estimated to be 1 with a sensitivity of $1(95 \% \mathrm{CI}=0.847$, 1 ) and a specificity of $1(95 \% \mathrm{CI}=0.717,1)$ (online suppl. Fig. 5).

Follow-up data were available for all patients from a minimum of 3 months to a maximum of 24 months. All the patients in the first group remained in clinical remission ( $<5$ for HBI and $0-1$ for PMSI) at follow-up with clinical scores superimposable to those postinduction and FL levels (measured at the reported follow-up times [Table 2] within a week after drug administration) reduced on the average by $93.5 \pm 9.8 \%$ (67-99.9) compared to baseline values. 
Table 2. FL and induction with biologic agents: management and follow-up

\begin{tabular}{|c|c|c|c|c|c|c|c|c|c|c|}
\hline $\mathrm{Pt}$ \# & $\begin{array}{l}\text { Disease } \\
\text { type }\end{array}$ & $\begin{array}{l}\text { Biologic } \\
\text { naïve }\end{array}$ & $\begin{array}{l}\text { Baseline } \\
\mathrm{FL}, \\
\mu \mathrm{g} / \mathrm{mL}\end{array}$ & $\begin{array}{l}\text { FL after first } \\
\text { induction dose, } \\
\mu \mathrm{g} / \mathrm{mL}\end{array}$ & $\begin{array}{l}\text { FL after second } \\
\text { induction dose, } \mu \mathrm{g} / \\
\mathrm{mL}\end{array}$ & Medication* & $\begin{array}{l}\text { HBI } \\
\text { (pre/ } \\
\text { postinduction) })^{+}\end{array}$ & $\begin{array}{l}\text { PMSI } \\
\text { (pre/ } \\
\text { postinduction) }^{+}\end{array}$ & Change in care & $\begin{array}{l}\text { Follow-up, clinical outcome (index) and } \\
\text { FL, } \mu \mathrm{g} / \mathrm{mL}\end{array}$ \\
\hline \multicolumn{11}{|c|}{ Responders } \\
\hline 1 & $\mathrm{CD}$ & Yes & 337 & 47 & 27 & Infliximab & $8 / 0$ & na & None & 12 months. Clinical remission ${ }^{\wedge}(0) .6 .25$ \\
\hline 2 & $\mathrm{CD}$ & Yes & 4,262 & 483 & 6.25 & Infliximab & $14 / 2$ & na & None & 24 months. Clinical remission (0). 6.25 \\
\hline 3 & UC & No & 1,306 & 82 & 6.25 & Infliximab & na & $6 / 0$ & None & 11 months. Clinical remission (1). 62 \\
\hline 4 & $\mathrm{CD}$ & Yes & 68 & 6.25 & 6.25 & Infliximab & $13 / 0$ & na & None & 10 months. Clinical remission (0). 6.25 \\
\hline 5 & UC & No & 386 & 173 & 94 & Infliximab & na & $9 / 1$ & None & 6 months. Clinical remission (0). 6.25 \\
\hline 6 & UC & No & 753 & 283 & 15 & Vedolizumab & na & $5 / 0$ & None & 12 months. Clinical remission (0). 6.25 \\
\hline 7 & $\mathrm{CD}$ & Yes & 159 & 51 & 27 & Adalimumab & $8 / 2$ & na & None & 12 months. Clinical remission (2). 23 \\
\hline 8 & $\mathrm{CD}$ & Yes & 229 & 6.25 & 6.25 & Infliximab & $14 / 1$ & na & None & 6 months. Clinical remission (1). 6.25 \\
\hline 9 & UC & Yes & 454 & 6.25 & 6.25 & Vedolizumab & na & $5 / 0$ & None & 8 months. Clinical remission (0). 6.25 \\
\hline 10 & $\mathrm{CD}$ & No & 43 & 6.25 & 6.25 & Adalimumab & $12 / 3$ & na & None & 6 months. Clinical remission (0). 6.25 \\
\hline 11 & UC & No & 2,585 & 44 & 32 & Infliximab & na & $5 / 0$ & None & 9 months. Clinical remission (1). 65 \\
\hline 12 & $\mathrm{CD}$ & No & 181 & 38 & 20 & Infliximab & $13 / 1$ & na & None & 8 months. Clinical remission (2). 61 \\
\hline 13 & $\mathrm{CD}$ & Yes & 819 & 23 & 14 & Adalimumab & $12 / 0$ & na & None & 7 months. Clinical remission (0). 6.25 \\
\hline 14 & $\mathrm{CD}$ & No & 597 & 77 & 8 & Adalimumab & $11 / 1$ & na & None & 3 months. Clinical remission (0). 6.25 \\
\hline 15 & $\mathrm{CD}$ & No & 227 & 6.25 & 6.25 & Infliximab & $8 / 0$ & na & None & 12 months. Clinical remission (0). 6.25 \\
\hline 16 & UC & Yes & 673 & 299 & 280 & Vedolizumab & na & $8 / 0$ & None & 6 months. Clinical remission (0). 7.3 \\
\hline 17 & UC & No & 600 & 217 & 59 & Infliximab & na & $8 / 1$ & None & 3 months. Clinical remission (0). 6.25 \\
\hline 18 & $\mathrm{CD}$ & Yes & 228 & 6.25 & 6.25 & Infliximab & $10 / 1$ & na & None & 18 months. Clinical remission (0). 6.25 \\
\hline \multicolumn{11}{|c|}{ Nonresponders } \\
\hline 19 & UC & No & 1,130 & 46 & 732 & Vedolizumab & na & $5 / 5$ & Therapy change & $\begin{array}{l}4 \text { months. Failed } 2 \text { more biologics (5). } \\
\text { Considering surgery }\end{array}$ \\
\hline 20 & UC & Yes & 3,811 & 775 & 3,529 & Infliximab & na & $8 / 7$ & $\begin{array}{l}\text { Total abdominal } \\
\text { colectomy }\end{array}$ & 4 months. Surgical remission (1) \\
\hline 21 & UC & Yes & 5,851 & 219 & 3,143 & Infliximab & na & $9 / 8$ & $\begin{array}{l}\text { Total abdominal } \\
\text { colectomy }\end{array}$ & 6 months. Surgical remission (1) \\
\hline 22 & UC & No & 1,423 & 1,155 & 2,140 & Infliximab & na & $5 / 5$ & Therapy change & $\begin{array}{l}3 \text { months. Currently in remission with } \\
\text { ustekinumab (1) }\end{array}$ \\
\hline 23 & UC & Yes & 277 & 248 & 386 & Vedolizumab & na & $6 / 6$ & Therapy change & $\begin{array}{l}3 \text { months. Currently in remission with } \\
\text { ustekinumab (1) }\end{array}$ \\
\hline 24 & UC & No & 3,637 & 927 & 2,805 & Vedolizumab & na & $9 / 7$ & Therapy change & $\begin{array}{l}3 \text { months. Currently in remission with } \\
\text { infliximab (1) }\end{array}$ \\
\hline 25 & $\mathrm{CD}$ & No & 221 & 190 & 357 & Infliximab & $16 / 14$ & na & $\begin{array}{l}\text { Small bowel } \\
\text { resection }\end{array}$ & 7 months. Surgical remission (2) \\
\hline 26 & $\mathrm{CD}$ & Yes & 936 & 151 & 442 & Certolizumab & $12 / 10$ & na & - & 3 months. (9) considering surgery \\
\hline 27 & UC & Yes & 2,700 & 433 & 2,447 & Vedolizumab & na & $6 / 6$ & - & 3 months. (5). Surgery scheduled \\
\hline
\end{tabular}

FL, fecal lactoferrin; HBI, Harvey-Bradshaw Index; PMSI, Partial Mayo Scoring Index; UC, ulcerative colitis; CD, Crohn's disease; na, not applicable. * Infliximab and vedolizumab are administered as 3 induction infusions at weeks 0,2 , and 6 ; adalimumab as 2 injections at weeks 0 and 2 ; and certolizumab as 3 injections at weeks 0,2 , and $4 .{ }^{+}$Pre/postinduction: before starting therapy and at the end of the induction. ${ }^{\wedge}$ Clinical remission defined as a score $<5$ (HBI) or $0-1$ (PMSI).

By contrast, of the 9 patients who did not respond to induction with rebounding FL, 4 were reinduced with new biologics (with 3 of them responding) while the others either underwent immediate surgery or are planning to undergo surgery due to previous or new multiple biologic failures.

Fecal Lactoferrin and Primary Nonresponse

\section{Discussion}

LOR and PNR to biologic agents represent an important and frequent event in IBD $[1,2]$. Symptomatic improvement after 12 weeks of initial biologic induction has been traditionally used to evaluate response to therapy [ 1 , 


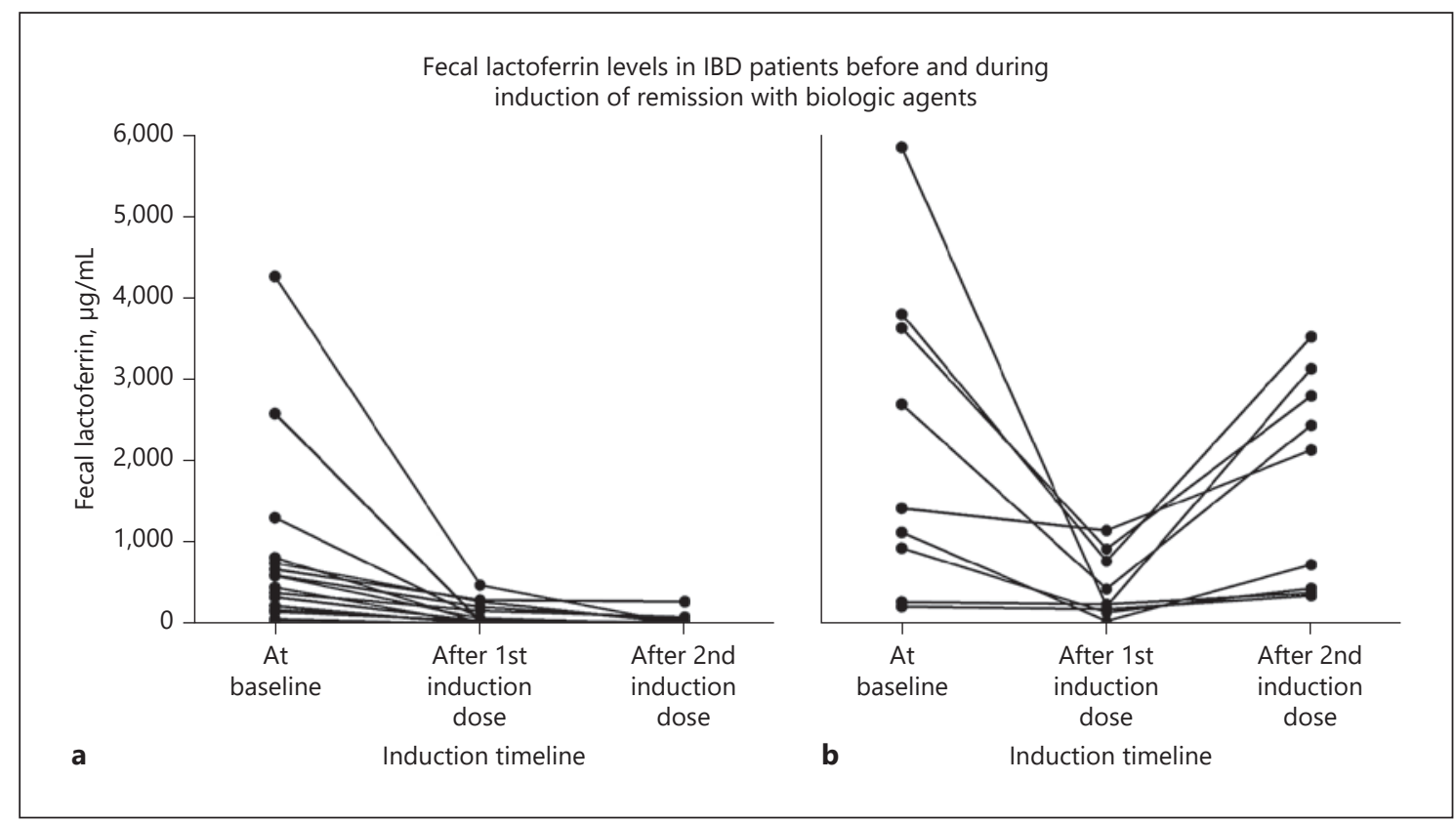

Fig. 1. FL levels in IBD patients at baseline (before therapy) and during biologic induction. a Patients showing a progressive FL decrease. These patients showed clinical response after induction and continued to be in full remission at follow-up. b Patients showing an initial FL drop with a subsequent increase. These patients did not respond clinically to induction, and therapy was changed to a different biologic or they underwent surgery. FL, fecal lactoferrin.

2]. More recently, proactive TDM has been proposed as a tool to optimize initial drug treatment in these patients [4-6]. With proactive TDM, target TL are stipulated before induction and - when measured and found subtherapeutic (in the absence of ADA) during and after induction - the drug dose is progressively increased. A number of studies have indeed shown that this interesting strategy could reduce PNR incidence and improve long-term outcomes [4-6]. However, solid evidence for routine clinical use is still lacking $[12,13]$. In addition, this strategy is expensive and has some practical shortcomings [7-9, 27], in particular in the USA [11]. One of the issues with this strategy is the assumption that there is a universal therapeutic TL that can be applied to all patients. However, establishing universal, practically useful therapeutic TL has been proven challenging since TL vary according to a number of factors [9], most importantly the individual disease burden [14]. Indeed, using a priori stipulated values, a substantial proportion of patients on biologics are either in remission with "subtherapeutic" TL or might experience PNR/LOR with "therapeutic" TL $[7,9,14,16,19$, $27,28]$.

A more practical approach to manage patients starting a biologic would be to evaluate whether the medication directly impacts the disease activity - that is, whether it is effective in reducing inflammation in the individual patient. Fecal markers of inflammation (FL/FC) accurately reflect mucosa inflammation $[17,29,30]$ and disease burden $[20,31]$ in IBD. However, their optimal use in disease management remains unclear [18]. Previous data obtained with FC [19] show that fecal markers timely mirror drug-mediated changes in inflammation since they directly reflect neutrophil translocation to the mucosa of the GI tract - a process quickly modulated by the activity of the inflammatory process [20]. Hence, fecal markers could potentially be used to immediately monitor biologic response in IBD. In our center, we have used this strategy over the years to assess patients with LOR to biologics and to monitor primary response during induction. We have reported here the data related to the latter group of patients.

We show that in all patients undergoing induction, the biologic agent induced an initial drop in FL compared to baseline levels. However, after the following dose, FL levels bounced back in approximately $30 \%$ of patients while keeping on decreasing (or remain low) in the others. Only in the latter patients clinical scores significantly dropped into the remission range at the end of induction. In the 
former group, the symptoms persisted and the medication was changed or patients underwent (or are planning) surgery if they had failed multiple biologics. Follow-up confirmed persistence of clinical remission (and FL levels reduced on the average by $94 \%$ ) over time only in initial responders, all of whom had 2 consecutive decreased FL levels during induction. Of the unresponsive patients who changed medication, 3 out of 4 responded to new biologics while the others - due to multiple biologic failures - either underwent immediate surgery or are planning to undergo surgery.

Logistic regression analysis showed that the 2 consecutively decreased FL values after the initial induction doses could predict remission at end of induction. When FL measurements were extended to the third induction dose, the patterns described above (continuous FL decrease vs. initial decrease with subsequent rebound) did not appear to change in individual patients (D. Sorrentino, unpublished). Hence, testing patients only after the second and the third induction dose could be equally informative. Models targeting single FL values (second FL value compared to baseline and second FL value compared to the first) were also able to predict remission. Models using the baseline FL or the FL drop after the first drug dose were less accurate in predicting remission.

Pavlidis et al. [32] have also shown that early postinduction changes in FC predict PNR clinical outcomes in $\mathrm{CD}$. By contrast to us, those authors tested the fecal marker up to 10 weeks after the first induction dose and only studied anti-TNF naïve patients, with more than half of them being on combination therapy. Similarly to us, De Vos et al. [33] have shown that a constant and deep decrease in FC during and after induction predicts remission at week 10 in patients treated with infliximab. Theede et al. [34] tested for FC 16 patients with active UC being treated with steroids and showed that the stool markers dropped immediately in all patients and correlated well with clinical and biochemical parameters. By contrast, Toyonaga et al. [35] in 27 UC patients (mostly treated with steroids with a minority treated with anti-TNF agents or tacrolimus) showed that clinical scores dropped faster than FC levels; this apparently due to daily FC variability. In our study, as discussed above, 2 consecutive FL drops as well as single FL values during induction predicted the response to therapy. These findings are consistent with the traditional clinical approach whereby the lack of clinical improvement after 2 induction doses was considered an indication to switch therapy $[1,2]$.

The significant drop (up to 98\%) in FL levels in all of our patients (including nonresponders) after the initial infusion/injection was somewhat unexpected. Billiet et al. [16] have reported a similar phenomenon testing different cytokines. It is possible that neutralizing antibodies produced after the first drug dose hamper response to the following drug administration [36]. However, it is also possible that inflammation switches to a different "driver" once a given pathway has been blocked by the medication in susceptible individuals $[14,37]$. It is also possible that patients with high inflammatory burden are more likely to experience PNR and might need a higher dose [16]. However, there is no clear evidence that initial induction doses might be insufficient in a proportion of patients due to a very high disease burden. Billiet et al. [16] suggested that some of the nonresponders might have lower tissue concentrations of medication and that - by inference - higher doses could be effective. In theory, it is possible that in our nonresponsive patients inflammation (and FL) increased after an initial drop due to the excessive consumption of medication because of the large disease burden. Indeed, baseline FL levels were higher on the average in nonresponsive patients. However, there was a large overlap among responders and nonresponders and a number of patients with very high baseline FL levels did respond to the same medications/doses/therapeutic intervals used in nonresponders.

An additional issue partially addressed by our study relates to the concept that anti-integrin therapies such as vedolizumab have a slow onset of action [38]. In our study there were both responders and nonresponders among patients on vedolizumab, irrespective of their previous experience with biologics. Hence, our limited observations suggest that these medications might actually act as fast as any other biologic agent.

Our study has some limitations. First of all, the sample size was relatively small and this might have affected our absolute remission rates and FL cutoff values. Replication of this study by others and on a larger scale would clearly be important. Nevertheless, the basic findings of this study (the high accuracy of early stool marker measures to predict response after induction) are fully consistent with those of others [32-35].

Also, our study is retrospective. In addition, we did not measure TL and ADA, which could have added interesting mechanistic information to our findings. A number of other issues are also worth a mention. First, adopting this fecal marker-based strategy might be challenging when the disease only involves a short small bowel segment and FL levels might [20] or might not [39] be elevated even in the absence of therapy. In general, detectable FL levels at baseline would be the only prerequisite 
to apply this strategy. Second, FL should be measured at the same, fixed time after each drug administration since it tends to increase over time [19] - for example, values measured 1 day after drug administration would be lower than those measured 10 days after [19]. Finally, it is important to keep in mind that steroids or any other effective fast-acting medications given before/during induction and the double FL testing could affect the results. In our patients, no concomitant medications were given in such period. If clinically needed (i.e., patient experiencing severe symptoms while waiting for induction), steroids could be initiated and kept on a stable dose well before and after induction - an approach that is classically used in clinical trials. In such case, baseline FL values would be those measured before induction with the patient already on stable steroid therapy.

In conclusion, the timely sensitivity of FL to drug-generated changes in inflammation provides the rationale for an accurate, rapid, and inexpensive stool marker-based strategy to predict PNR in IBD patients undergoing induction with biologic agents. We have recently shown that such fecal marker-based approach might also be applied to patients experiencing a potential LOR to biologic agents [40]. However, larger prospective studies will be needed to evaluate the potential applicability of these strategies to clinical practice and to explore any potential complementary role for TDM [41].

\section{Acknowledgements}

We thank James M. Gray for technical help and James Boone for critically reviewing the manuscript.

\section{Statement of Ethics}

There was no direct patient involvement. The study conformed to the guidelines of the 1975 Declaration of Helsinki as reflected in a priori approval by Carilion Clinic Ethics Committee ("Utilization of stool markers to manage IBD patients losing response to biologics"- approval \#IRB-18-259). No patient consent was deemed necessary by the Ethics Committee under 45 CFR $46.116(d)$.

\section{Conflict of Interest Statement}

Dario Sorrentino has received consulting fees from Abbott/AbbVie, Schering-Plough, MSD, Janssen Research \& Development, LLC., Centocor Inc., TechLab, Hoffmann-LaRoche, Giuliani, Schering-Plough, and Ferring; research grants from AbbVie, Janssen Research \& Development, LLC, Schering-Plough, TechLab, Centocor, Takeda; and serves in the Speakers Bureau of AbbVie and the National Faculty of Janssen. Vu Nguyen has received grant support from AbbVie Inc. Kim Love has no conflicts of interest to declare.

\section{Funding Sources}

This research received no specific grant from any funding agency in the public, commercial, or not-for-profit sectors.

\section{Author Contributions}

Study concept and design: D.S.; acquisition of data: D.S. and V.Q.N.; analysis and interpretation of data: D.S. and K.L.; drafting of the manuscript: D.S. and K.L.; revision of the manuscript for important intellectual content: D.S., V.Q.N., and K.L.; approval of the final manuscript: D.S., V.Q.N., and K.L.; guarantor of the article: D.S.

\section{References}

1 Danese S, Fiorino G, Reinisch W. Review article: causative factors and the clinical management of patients with Crohn's disease who lose response to anti-TNF-alpha therapy. Aliment Pharmacol Ther. 2011;34:1-10.

2 Gisbert JP, Panés J. Loss of response and requirement of infliximab dose intensification in Crohn's disease: a review. Am J Gastroenterol. 2009;104(3):760-7.

3 Vande Casteele N, Herfarth H, Katz J, FalckYtter Y, Singh S. American gastroenterological association institute technical review on the role of therapeutic drug monitoring in the management of inflammatory bowel diseases. Gastroenterology. 2017;153(3):835-e6.
4 Fernandes SR, Bernardo S, Simões C, Gonçalves $A R$, Valente A, Baldaia C, et al. Proactive infliximab drug monitoring is superior to conventional management in inflammatory bowel disease. Inflamm Bowel Dis. 2020;26(2):263-70.

5 Sánchez-Hernández JG, Rebollo N, MartinSuarez A, Calvo MV, Muñoz F. A 3-year prospective study of a multidisciplinary early proactive therapeutic drug monitoring programme of infliximab treatments in inflammatory bowel disease. Br J Clin Pharmacol. 2020;86(6):1165-75.

6 Papamichael K, Chachu KA, Vajravelu RK, Vaughn BP, Ni J, Osterman MT, et al. Improved long-term outcomes of patients with inflammatory bowel disease receiving proactive compared with reactive monitoring of serum concentrations of infliximab. Clin Gastroenterol Hepatol. 2017;15(10):1580-e3.
7 Pariente B, Pineton de Chambrun G, Krzysiek R, Desroches M, Louis G, De Cassan C, et al. Trough levels and antibodies to infliximab may not predict response to intensification of infliximab therapy in patients with inflammatory bowel disease. Inflamm Bowel Dis. 2012; 18(7):1199-206.

8 Ungar B, Chowers $\mathrm{Y}$, Yavzori M, Picard O, Fudim E, Har-Noy O, et al. The temporal evolution of antidrug antibodies in patients with inflammatory bowel disease treated with infliximab. Gut. 2014;63(8):1258-64.

9 Gibson DJ, Ward MG, Rentsch C, Friedman AB, Taylor KM, Sparrow MP, et al. Review article: determination of the therapeutic range for therapeutic drug monitoring of adalimumab and infliximab in patients with inflammatory bowel disease. Aliment Pharmacol Ther. 2020 Mar;51(6):612-28. 
10 Ricciuto A, Dhaliwal J, Walters TD, Griffiths AM, Church PC. Clinical outcomes with therapeutic drug monitoring in inflammatory bowel disease: a systematic review with metaanalysis. J Crohns Colitis. 2018;12(11):130215.

11 Grossberg LB, Papamichael K, Feuerstein JD, Siegel CA, Ullman TA, Cheifetz AS. A survey study of gastroenterologists' attitudes and barriers toward therapeutic drug monitoring of anti-TNF therapy in inflammatory bowel disease. Inflamm Bowel Dis. 2017;24(1):1917.

12 Shah R, Hoffman GR, El-Dallal M, Goldowsky AM, Chen Y, Feuerstein JD. Is therapeutic drug monitoring for anti-tumor necrosis factor agents in adults with inflammatory bowel disease ready for standard of care?: A systematic review and meta-analysis. J Crohns Colitis. 2020 Sep 7;14(8):1057-65.

13 Adamina M, Bonovas S, Raine T, Spinelli A, Warusavitarne J, Armuzzi A, et al. ECCO guidelines on therapeutics in Crohn's disease: surgical treatment. J Crohns Colitis. 2020; 14(2):155-68

14 Sorrentino D, Nguyen V, Henderson C, Bankole A. Therapeutic drug monitoring and clinical outcomes in immune mediated diseases: the missing link. Inflamm Bowel Dis. 2016;22(10):2527-37.

15 Roblin X, Boschetti G, Duru G, Williet N, Deltedesco E, Phelip JM, et al. Distinct thresholds of infliximab trough level are associated with different therapeutic outcomes in patients with inflammatory bowel disease: a prospective observational study. Inflamm Bowel Dis. 2017;23(11):2048-53.

16 Billiet T, Cleynen I, Ballet V, Claes K, Princen F, Singh S, et al. Evolution of cytokines and inflammatory biomarkers during infliximab induction therapy and the impact of inflammatory burden on primary response in patients with Crohn's disease. Scand J Gastroenterol. 2017;52(10):1086-92.

17 D'Incà R, Caccaro R. Measuring disease activity in Crohn's disease: what is currently available to the clinician. Clin Exp Gastroenterol. 2014;7:151-61.

18 Boon GJ, Day AS, Mulder CJ, Gearry RB. Are faecal markers good indicators of mucosal healing in inflammatory bowel disease? World J Gastroenterol. 2015;21(40):1146980.

19 Sorrentino D, Marino M, Dassopoulos T, Zarifi D, Del Bianco T. Low dose infliximab for prevention of postoperative recurrence of Crohn's disease: long term follow-up and impact of infliximab trough levels and antibodies to infliximab. PLoS One. 2015;15(10): e0144900.
20 Rubio MG, Amo-Mensah K, Gray JM, Nguyen VQ, Nakat S, Grider D, et al. Fecal lactoferrin accurately reflects mucosal inflammation in inflammatory bowel disease. World J Gastrointest Pathophysiol. 2019;10(5):54-63.

21 Benevento G, Avellini C, Terrosu G, Geraci M, Lodolo I, Sorrentino D. Diagnosis and assessment of Crohn's disease: the present and the future. Expert Rev Gastroenterol Hepatol. 2010;4(6):757-66.

22 Langhorst J, Boone J. Fecal lactoferrin as a noninvasive biomarker in inflammatory bowel diseases. Drugs Today. 2012;48(2): 149-61.

23 Peyrin-Biroulet L, Panés J, Sandborn WJ, Vermeire S, Danese S, Feagan BG, et al. Defining disease severity in inflammatory bowel diseases: current and future directions. Clin Gastroenterol Hepatol. 2016;14(3):348-e17.

24 Lewis JD, Chuai S, Nessel L, Lichtenstein GR, Aberra FN, Ellenberg JH. Use of the noninvasive components of the Mayo score to assess clinical response in ulcerative colitis. Inflamm Bowel Dis. 2008;14(12):1660-6.

25 D'Haens G, Sandborn WJ, Feagan BG, Geboes $\mathrm{K}$, Hanauer SB, Irvine EJ, et al. A review of activity indices and efficacy end points for clinical trials of medical therapy in adults with ulcerative colitis. Gastroenterology. 2007; 132(2):763-86.

26 Vermeire S, Schreiber S, Sandborn WJ, Dubois C, Rutgeerts P. Correlation between the Crohn's disease activity and Harvey-Bradshaw indices in assessing Crohn's disease severity. Clin Gastroenterol Hepatol. 2010;8(4): 357-63.

27 Magro F, Afonso J, Lopes S, Coelho R, Goncalves R, Caldeira P, et al. Calprotectin and the magnitude of antibodies to infliximab in clinically-stable ulcerative colitis patients are more relevant than infliximab trough levels and pharmacokinetics for therapeutic escalation. EBioMedicine. 2017;21:123-30.

28 Chaparro M, Guerra I, Iborra M, Cabriada JL, Bujanda L, Taxonera C, et al. Usefulness of monitoring antitumor necrosis factor serum levels during the induction phase in patients with Crohn's disease. Eur J Gastroenterol Hepatol. 2020;32(5):588-96.

29 Pauwels RWM, de Vries AC, van der Woude CJ. Fecal calprotectin is a reliable marker of endoscopic response to vedolizumab therapy: a simple algorithm for clinical practice. J Gastroenterol Hepatol. 2020;35(11):1893-901.

30 El-Matary W, Abej E, Deora V, Singh H, Bernstein CN. Impact of fecal calprotectin measurement on decision-making in children with inflammatory bowel disease. Front Pediatr. 2017 Jan 25;5:7.
31 Dai C, Jiang M, Sun MJ, Cao Q. Fecal lactoferrin for assessment of inflammatory bowel disease activity: a systematic review and metaanalysis. J Clin Gastroenterol. 2020;54(6): 545-53.

32 Pavlidis P, Gulati S, Dubois P, Chung-Faye G, Sherwood R, Bjarnason I, et al. Early change in faecal calprotectin predicts primary nonresponse to anti-TNFa therapy in Crohn's disease. Scand J Gastroenterol. 2016;51(12): 1447-52.

33 De Vos M, Dewit O, D’Haens G, Baert F, Fontaine F, Vermeire $S$, et al. Fast and sharp decrease in calprotectin predicts remission by infliximab in anti-TNF naïve patients with ulcerative colitis. J Crohns Colitis. 2012;6(5): 557-62.

34 Theede K, Kiszka-Kanowitz M, Nielsen AM, Nordgaard-Lassen I. The correlation between fecal calprotectin, simple clinical colitis activity index and biochemical markers in ulcerative colitis during high-dose steroid treatment. Scand J Gastroenterol. 2014;49:418-23.

35 Toyonaga T, Kobayashi T, Nakano M, Saito E, Umeda S, Okabayashi S, et al. Usefulness of fecal calprotectin for the early prediction of short-term outcomes of remission-induction treatments in ulcerative colitis in comparison with two-item patient-reported outcome. PLoS One. 2017;12:e0185131.

36 Papamichael K, Gils A, Rutgeerts P, Levesque BG, Vermeire S, Sandborn WJ, et al. Role for therapeutic drug monitoring during induction therapy with TNF antagonists in IBD: evolution in the definition and management of primary nonresponse. Inflamm Bowel Dis. 2015;21(1):182-97.

37 Barber GE, Yajnik V, Khalili H, Giallourakis C, Garber J, Xavier R, et al. Genetic markers predict primary non-response and durable response to anti-TNF biologic therapies in Crohn's disease. Am J Gastroenterol. 2016; 111(12):1816-22.

38 Vasudevan A, Gibson PR, van Langenberg DR. Time to clinical response and remission for therapeutics in inflammatory bowel diseases: what should the clinician expect, what should patients be told? World J Gastroenterol. 2017;23(35):6385-402.

39 Sorrentino D, Nguyen VQ. Clinically significant small bowel Crohn's disease might only be detected by capsule endoscopy. Inflamm Bowel Dis. 2018;24(7):1566-74.

40 Sorrentino D, Gray JM. Timely monitoring of inflammation by fecal lactoferrin rapidly predicts therapeutic response in inflammatory bowel disease. Inflamm Bowel Dis. 2021 Jan 27;izaa348.

41 Dreesen E, Baert F, Laharie D, Bossuyt P, Bouhnik Y, Buisson A, et al. Monitoring a combination of calprotectin and infliximab identifies patients with mucosal healing of Crohn's disease. Clin Gastroenterol Hepatol. 2020;18(3):637-e11. 